\title{
Tetrahydroxystilbene glucoside protects against LPS-induced endothelial dysfunction via inhibiting RhoA/ROCK signaling and F-actin remodeling
}

\author{
Yan $\mathrm{Qi}^{1, \star}$, Xiuying Liang ${ }^{1, \star}$, Xia Hu${ }^{1}$, Haiyan $\mathrm{He}^{2}$, Lian Tang ${ }^{3}$ and Wenjuan $\mathrm{Yao}^{1,4}$ \\ ${ }^{1}$ School of Pharmacy, Nantong University, Nantong, China \\ ${ }^{2}$ Department of Respiratory medicine, Nantong First People's Hospital, Second Affiliated Hospital of Nantong University, \\ Nantong, China \\ ${ }^{3}$ Pharmacy Department, Nantong First People's Hospital, Second Affiliated Hospital of Nantong University, Nantong, China \\ ${ }^{4}$ Key Laboratory of Inflammation and Molecular Drug Target of Jiangsu Province, Nantong, China
}

\begin{abstract}
We analyzed the role of the RhoA/ROCK pathway in regulating endothelial dysfunction triggered by LPS and the protective effects of TSG $(2,3,5,4$ '-tetrahydroxystilbene-2-O- $\beta$-Dglucoside). Human umbilical vein endothelial cells (HUVECs) were treated with LPS at different concentrations or at different time points. Cells were also pretreated with $30 \mu \mathrm{M}$ ROCK inhibitor Y27632 for $30 \mathrm{~min}$ or different concentrations of TSG for $24 \mathrm{~h}$ and then were incubated with $100 \mu \mathrm{g} / \mathrm{ml}$ LPS for another $24 \mathrm{~h}$. The results showed that LPS treatment significantly reduced endothelial cell viability, increased LDH release, and promoted cell necrosis in a dose- and time-dependent manner, which was dramatically inhibited by TSG pretreatment. Furthermore, LPS induction significantly enhanced the expression of RhoA, ROCK1, and ROCK2 and the activation of ROCK; these effects were reduced by TSG pretreatment. The suppression of either RhoA or ROCK significantly improved LPS-induced endothelial cell viability, and reduced cell necrosis and LDH release. In addition, LPS treatment promoted F-actin skeleton rearrangement and contraction ring formation around the plasma membrane, which was greatly inhibited by the suppression of the RhoA/ROCK pathway or TSG pretreatment. In conclusion, TSG may inhibit F-actin cytoskeletal remodeling by blocking RhoA/ROCK signaling and thus reduce LPS-induced endothelial cell toxicity.
\end{abstract}

Key words: Tetrahydroxystilbene glucoside - RhoA/ROCK pathway - LPS - Actin - Endothelial dysfunction

\begin{abstract}
Abbreviations: DAPI, 4'6-diamidino-2-phenylindole; DMSO, dimethylsulfoxide; FBS, fetal bovine serum; FITC, fluorescein isothiocyanate; HUVECs, human umbilical vein endothelial cells; LDH, lactate dehydrogenase; LPS, lipopolysaccharide; MLC2, myosin light chain 2; MTT, 3-[4,5-dimethylthiazol-2-yl]-2,5-diphenyltetrazolium bromide; MYPT1, myosin phosphatase targeting subunit 1; PI, propidium iodide; ROCK, Rho-kinase; SDS, sodium dodecyl sulfate; TBST, Tris-buffered saline-Tween; TSG, 2,3,5,4'-tetrahydroxystilbene-2-O- $\beta$-D-glucoside.
\end{abstract}

\section{Introduction}

Lipopolysaccharide (LPS) is the main causative pathological agent of gram-negative bacilli. LPS in the blood is an impor-

\footnotetext{
* These authors contributed equally to this work.

Correspondence to: Wenjuan Yao, Department of Pharmacology, School of Pharmacy, Nantong University, 19 QiXiu Road, Nantong 226001, Jiangsu, China

E-mail: yaowenjuan0430@aliyun.com
}

tant circulatory pathophysiological characteristic of sepsis and septic shock (Fu et al. 2015). In addition, endotoxemia can cause multiple organ failure and many diseases, such as acute respiratory distress syndrome (ARDS), severe burns and trauma. LPS-induced injury of endothelial cells is an important event for the development of these diseases (Charbonney et al. 2016; Cheng et al. 2017; Straat et al. 2017; Tian et al. 2019). Therefore, revealing the mechanisms of LPS in endothelial cell dysfunction is important for the clinical treatment of endotoxemia. 
TSG (2,3,5,4'-tetrahydroxystilbene-2-O- $\beta$-D-glucoside) is the main bioactive component extracted from the dried tuber of the traditional Chinese medicine, Polygonum multiflorum. It has been reported that TSG has multiple pharmacological activities, such as anti-oxidation, antiinflammation, and anti-platelet aggregation, and can improve memory and learning ability (Büchter et al. 2015; Wu et al. 2017). Previous studies have also found that TSG inhibits matrix metalloproteinase activation, inflammatory responses, and the proliferation of vascular smooth muscle cells, and delays vascular and endothelial cell aging in atherosclerosis rats (Zhang et al. 2008; Han et al. 2012). However, the effects of TSG on LPS-induced endothelial cell dysfunction remain unknown.

Small Rho GTPases of the Rho family, mainly including RhoA, Cdc42 and Rac1, play a role in molecular switching in a series of intracellular activities by mediating the transformation between GDP-bound inactive forms and GTPbound active forms (Heasman and Ridley 2008). Rho-kinase (ROCK) is one of the best-characterized effectors of small GTPase RhoA and contains two similar isoforms, ROCK1 and ROCK2 (Matsui et al. 1996; Nakagawa et al. 1996). It has been reported that ROCK deletion attenuates diabetesinduced vascular endothelial dysfunction by preventing increased arginase activity, and by reducing nitric oxide production (Yao et al. 2013). ROCK kinases have roles in the phosphorylation of myosin light chain 2 (MLC2) and cofilin to modulate the actin cytoskeleton (Shi et al. 2013). However, it remains largely unclear whether the RhoA/ ROCK pathway or its regulation of the actin cytoskeleton is involved in LPS-mediated endothelial dysfunction. In this study, we investigated the specific role of the RhoA/ROCK pathway in LPS-induced F-actin cytoskeleton and endothelial dysfunction and further demonstrated the protective effects of TSG on cell injury induced by LPS.

\section{Materials and Methods}

\section{Materials}

TSG (purity above 98\%) was purchased from Sikehua Biosciences (\#2005119; Chengdu, China). DMEM medium and fetal bovine serum (FBS) were obtained from GibcoBRL (Grand Island, NY). 3-[4, 5-dimethylthiazol-2-yl]-2, 5-diphenyltetrazolium bromide (MTT), dimethylsulfoxide (DMSO), 4', 6-diamidino-2-phenylindole (DAPI), and sodium dodecyl sulfate (SDS) were purchased from Sigma Chemical Co. (St. Louis, MO). Primary antibodies for RhoA (\#2117), MYPT1 (\#2634), phospho-MYPT1 (Thr696 and Thr853) (\#5163 and \#4563), ROCK1 (\#4035), ROCK2 (\#8236), GAPDH (\#2118), and von Willebrand factor (\#65707) were obtained from Cell Signaling Technology
(Beverly, MA, USA). Horseradish peroxidase (HRP)conjugated anti-rabbit IgG (sc-2357) was purchased from Santa Cruz Biotechnology (Santa Cruz, CA, USA). The annexin V-FITC/PI double staining kit was purchased from Clontech (Mountain View, CA, USA). The LDH (lactate dehydrogenase) assay kit (ab197004) was obtained from Abcam (Cambridge, MA, USA). The specific ROCK inhibitor Y-27632 was purchased from Selleckchem (Houston, TX, USA). LPS was from Sigma (Saint Louis, MO, USA). siRNAs were synthesized by Sangon Gene Company (Shanghai, China). All of the other chemicals used in these experiments were of analytical grade and made in China.

\section{Cell culture and treatment}

Human umbilical vein endothelial cells (HUVECs) were isolated by collagenase digestion of umbilical veins from fresh cords. The study protocol was approved by the Ethics Review Committee of the Affiliated Hospital of Nantong University. HUVECs were cultured in DMEM (HG) with 10\% FBS in a humidified atmosphere of $5 \% \mathrm{CO}_{2}$ and $95 \%$ air at $37^{\circ} \mathrm{C}$, and passaged every $2-3$ days by trypsinization. HUVECs at passages 3-6 were used in this study and identified by von Willebrand factor (data not shown).

HUVECs were treated with different concentrations of LPS $(0,1,10,25,50$, and $100 \mu \mathrm{g} / \mathrm{ml})$ for $24 \mathrm{~h}$ or $100 \mu \mathrm{g} / \mathrm{ml}$ LPS at different time points $(0,6,12,24,48$, and $72 \mathrm{~h})$. The cells were pretreated with $30 \mu \mathrm{M}$ of the ROCK pan-inhibitor Y27632 for $30 \mathrm{~min}$ before being incubated with $100 \mu \mathrm{g} / \mathrm{ml}$ LPS for $24 \mathrm{~h}$. The cells were pretreated for $24 \mathrm{~h}$ with different concentrations of TSG $(0,10,25,50$, and $100 \mu \mathrm{M})$, and then exposed to $100 \mu \mathrm{g} / \mathrm{ml} \mathrm{LPS}$ for $24 \mathrm{~h}$.

\section{MTT assay}

Cell viability was measured by the MTT method. After treatments, the cells were added into $20 \mu \mathrm{l}$ MTT solution ( $5 \mathrm{mg} / \mathrm{ml}$ MTT in phosphate-buffered saline solution) and then incubated at $37^{\circ} \mathrm{C}$ for $4 \mathrm{~h}$. Subsequently, the MTTcontaining DMEM was removed and replaced by $150 \mu \mathrm{l}$ of DMSO, and then the plates were gently shaken for $10 \mathrm{~min}$. Absorbance was measured at $570 \mathrm{~nm}$ using a microplate reader. Cell viability was expressed as the fold of the value of the control culture.

\section{$L D H$ release assay}

Cell toxicity was further confirmed by measuring the activity of $\mathrm{LDH}$. An LDH release assay was performed according to the protocols of the LDH kit. Briefly, the culture medium was mixed with NAD and lactate solution (Xu et al. 2013). The absorbance of the samples was measured with a fluo- 
rescent microplate reader equipped with a filter for $\mathrm{Ex} / \mathrm{Em}$ $=535 / 587 \mathrm{~nm}$.

\section{Flow cytometry}

To detect cell apoptosis, the cells were washed with PBS and then stained with annexin $\mathrm{V}$-fluorescein isothiocyanate (FITC) and propidium iodide (PI) for $20 \mathrm{~min}$ according to the annexin V-FITC and PI double staining kit instructions. Briefly, cells were collected by centrifugation at $1000 \mathrm{rpm}$ for $5 \mathrm{~min}$ and then gently resuspended in $500 \mu$ of binding buffer. Then, $5 \mu \mathrm{l}$ of annexin V-FITC and $5 \mu \mathrm{l}$ of PI solution (final concentrations of both, $1 \mu \mathrm{g} / \mathrm{ml}$ ) were added and incubated with cells in the dark for $15 \mathrm{~min}$. At the end of incubation, the cells were and analyzed by flow cytometry (FACSCalibur, BD Biosciences, San Jose, CA, USA).

\section{Western blot analysis}

Cells after various treatments were lysed in protein lysis buffer (0.2\% SDS, 1\% NP-40, 5 mM EDTA, 1 mM PMSF, $10 \mu \mathrm{g} / \mathrm{ml}$ leupeptin, and $10 \mu \mathrm{g} / \mathrm{ml}$ aprotinin) and then centrifuged at $12,000 \mathrm{rpm}$ for $15 \mathrm{~min}$ at $4^{\circ} \mathrm{C}$. The protein concentrations were determined with the BCA-100 Protein Quantitative Analysis kit (Sangon, Shanghai, China). The protein samples were separated by $10 \%$ sodium dodecyl sulfate-polyacrylamide gel electrophoresis (SDS-PAGE) and then transferred onto a polyvinylidene fluoride (PVDF) membrane (Millipore, Billerica, MA, USA). The membranes were incubated at room temperature for $2 \mathrm{~h}$ with $5 \%$ skim (non-fat) milk in Tris-buffered saline-Tween (TBST). After washing with TBST, the membranes were then incubated with appropriate antibodies overnight at $4^{\circ} \mathrm{C}$. Subsequently, the membranes were washed with TBST and incubated with HRP-conjugated secondary antibodies for $1 \mathrm{~h}$ at room temperature. Band intensities were quantified by densitometry and imaging software (Labworks).

\section{Transfection of siRNA}

The siRNAs were a human RhoA-siRNA (sense: UGGCAGAUAUCGAGGUGGAdTdT; anti-sense: UCCACCUCGAUAUCUGCCAdTdT), a human ROCK1-siRNA (sense: GACGCAAGAAAGCAAGAAAdTdT; anti-sense: UUUCUUGCUUUCUUGCGUCdTd), and a human ROCK2-siRNA (sense: GGCACGACUAGCAGAUAAAdTdT; anti-sense: UUUAGCUGCUAGUCGUGCCdTdT). Synthetic siRNAs were dissolved into DEPC water at a concentration of $20 \mu \mathrm{M}$. The transfection reagent Lipofectamine 2000 and the siRNAs were diluted with Opti-MEM (Invitrogen, Karlsruhe, Germany) and incubated for $5 \mathrm{~min}$ at room temperature prior to use. Subsequently, the siRNAs solution and Lipofectamine 2000 solution were mixed and incubated for $20 \mathrm{~min}$ at room temperature to allow complex formation. The mixture was added to the HUVECs and incubated at $37^{\circ} \mathrm{C}$ with $5 \% \mathrm{CO}_{2}$ for $48 \mathrm{~h}$.

\section{F-actin imaging}

To detect F-actin, HUVECs were seeded and attached to gelatin-coated glass coverslips. The cells were fixed with $4 \%$ paraformaldehyde in PBS for 20 min and permeabilized with $0.2 \%$ Triton X-100 for $30 \mathrm{~min}$. After blocking with $5 \%$ bovine serum albumin, the cells were stained with rhodamineconjugated phalloidin (Life Technologies) for 20-90 min at room temperature. After washing with PBS, the cells were stained with DAPI for $20 \mathrm{~min}$. Stained cells were viewed under a fluorescent microscope equipped with appropriate filters (Nikon, Japan).

\section{Statistical analysis}

All experiments were repeated at least three times. Data were expressed as means $\pm \mathrm{SD}$. One-way ANOVA followed by Student's $t$-test was used for statistical analysis with SPSS.

\section{Results}

\section{The toxicological effects of LPS on HUVECs}

We added different concentrations of LPS $(0,1,10,25,50$, and $100 \mu \mathrm{g} / \mathrm{ml}$ ) to the cell culture media of HUVECs. After treatment for $24 \mathrm{~h}$, cell viability was assessed by MTT assay. As shown in Fig. 1A, the viability was significantly decreased after treatment with 25, 50, and $100 \mu \mathrm{g} / \mathrm{ml}$ LPS when compared with the control group $(0 \mu \mathrm{g} / \mathrm{ml} \mathrm{LPS})$, while the cell viability after treatment with 1 or $10 \mu \mathrm{g} / \mathrm{ml}$ LPS had no obvious changes. In addition, we also treated HUVECs with LPS at a final concentration of $100 \mu \mathrm{g} / \mathrm{ml}$ at different time points $(0,6,12,24,48$, and $72 \mathrm{~h})$. MTT results showed that the viability was significantly decreased after LPS treatment for 24, 48 and $72 \mathrm{~h}$ when compared with the control group $(0 \mathrm{~h})$. However, the cell viability was not significantly decreased when cells were treated with LPS at 6 and 12 h. Furthermore, we detected the cytotoxicity of LPS by measuring LDH release into the culture medium. As shown in Fig. 1B, LDH release was greatly increased after treatment with 25,50 , and $100 \mu \mathrm{g} / \mathrm{ml}$ LPS when compared with the control group, while 1 and $10 \mu \mathrm{g} / \mathrm{ml}$ LPS treatment did not affect the release of $\mathrm{LDH}$. The $\mathrm{LDH}$ release of HUVECs was dramatically enhanced after LPS treatment for 24, 48 and $72 \mathrm{~h}$, while no obvious changes were observed after LPS treatment for 6 and $12 \mathrm{~h}$. Finally, we detected the effect of LPS on endothelial cell apoptosis by performing 
Qi et al.

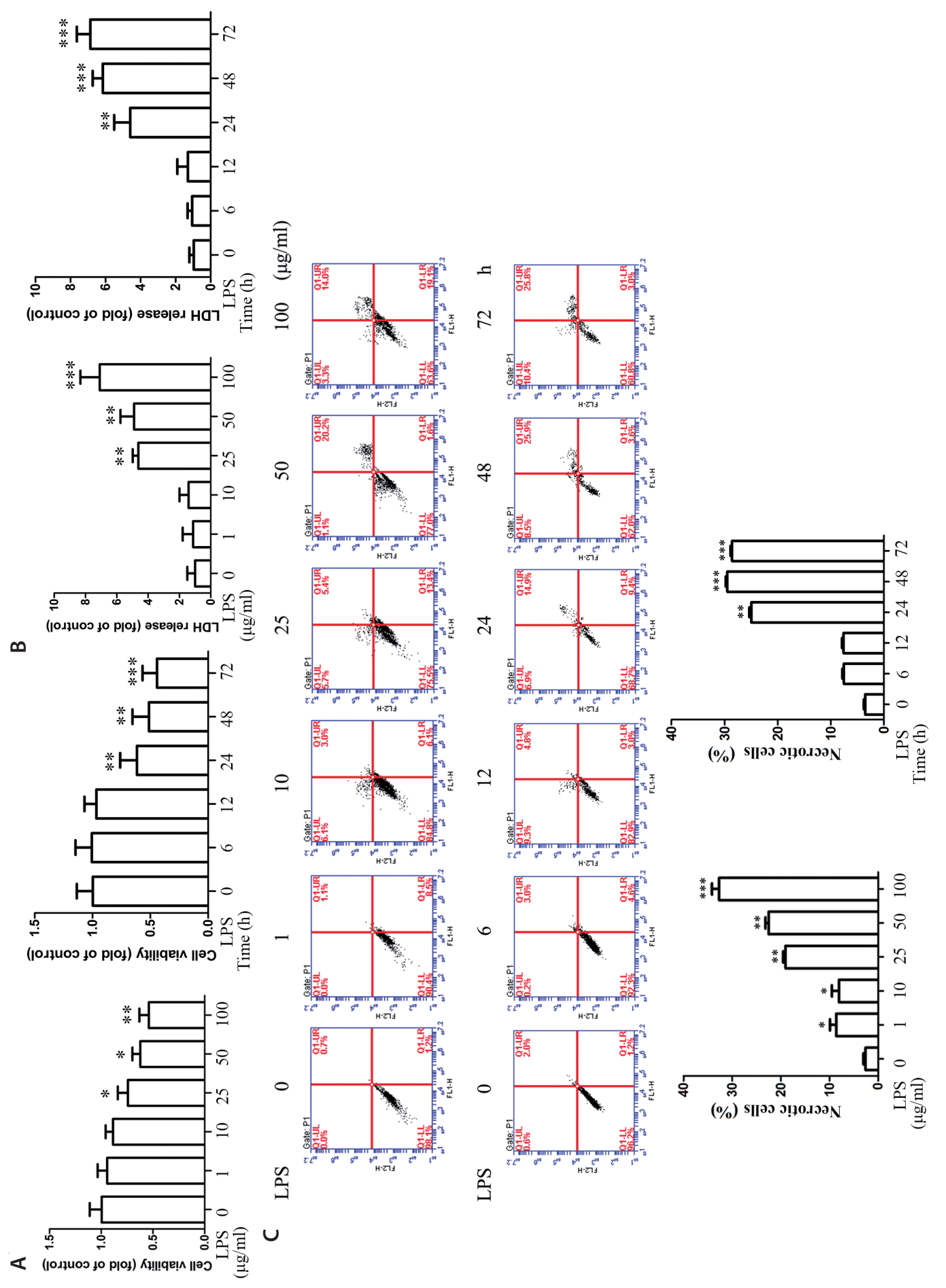

है 品 芩品क

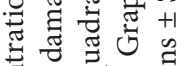

प्च च छे

定

我定

焉

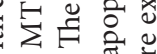

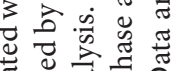

记

क्ष

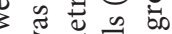

3 퀑

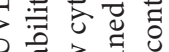

类

仓ु

\& 它青

在宝旸

중

के

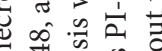

현렬

की

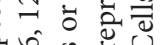

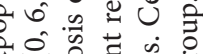

न

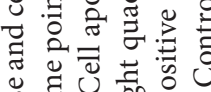

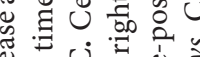

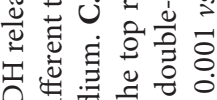

急寻导它

元

$\infty$ व

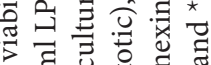

चु

5 8 需

击

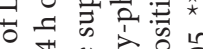

군

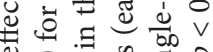

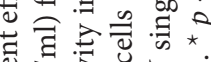

过

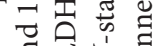
주

in .0 目

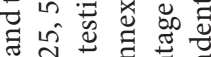

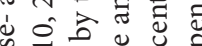

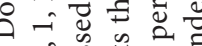

- 0 过

0 的

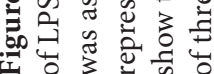


flow cytometry. As shown in Fig. 1C, compared with the control group, LPS treatment at different concentrations significantly promoted total cell apoptosis (including earlyphase apoptotic and late-phase apoptotic/necrotic cells) in a dose-dependent manner, in which late-phase apoptotic/ necrotic cells were dominant. Total cell necrosis was greatly increased after treatment with $100 \mu \mathrm{g} / \mathrm{ml} \mathrm{LPS} \mathrm{for} \mathrm{24,} 48$ and $72 \mathrm{~h}$. Since LDH release significantly increased, we think that endothelial cytotoxicity induced by LPS treatment was dominated by necrosis. Taken together, LPS treatment significantly promoted endothelial cell necrosis and reduced cell viability in a dose- and time-dependent manner. Since cell survival was less than $50 \%$ after prolonged exposure to
LPS, we performed $100 \mu \mathrm{g} / \mathrm{ml}$ LPS treatment for $24 \mathrm{~h}$ in subsequent experiments.

\section{Inhibitory effects of TSG on LPS-induced cytotoxicity in HUVECs}

HUVECs were pretreated with different concentrations of TSG $(10,25,50$, and $100 \mu \mathrm{M})$ for $24 \mathrm{~h}$ and then treated with LPS at a final concentration of $100 \mu \mathrm{g} / \mathrm{ml}$ for another $24 \mathrm{~h}$. MTT assays showed that $100 \mu \mathrm{M}$ TSG treatment alone exhibited no significant effects on cell viability in HUVECs. TSG pretreatment of 50 and $100 \mu \mathrm{M}$ significantly promoted cell viability after LPS treatment (Fig. 2A). As shown in Fig. 2B,
A

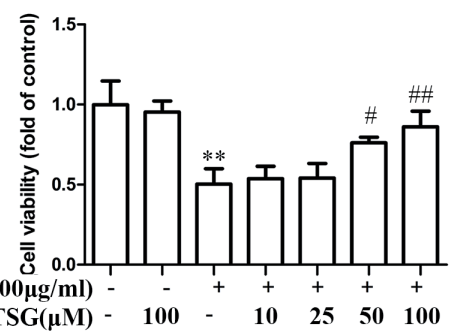

B

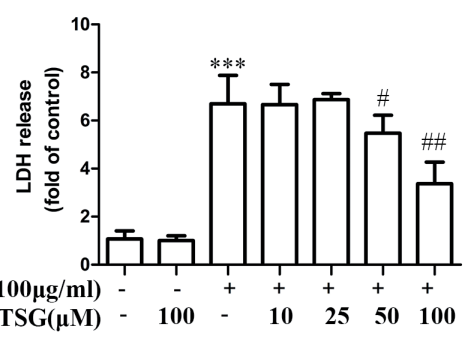

C

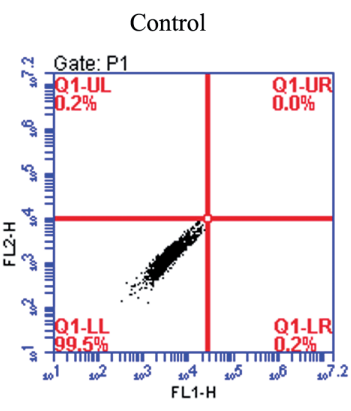

LPS $+10 \mu \mathrm{M} \mathrm{TSG}$
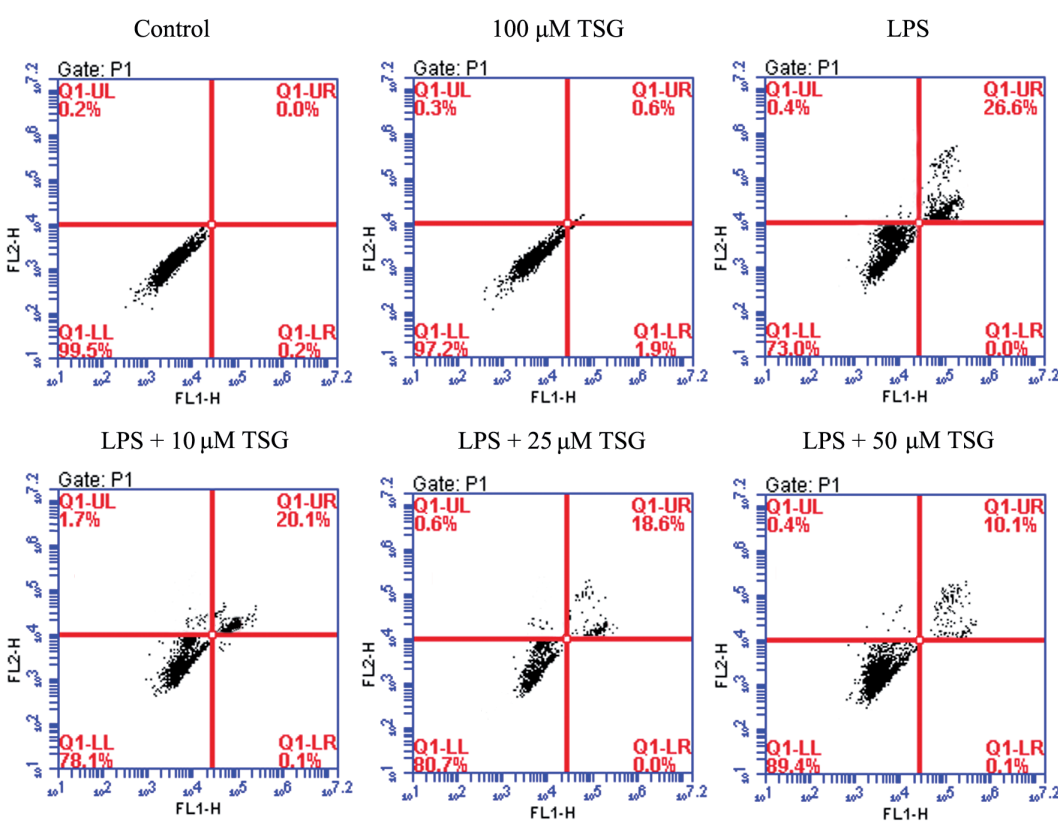

LPS $+25 \mu \mathrm{M}$ TSG

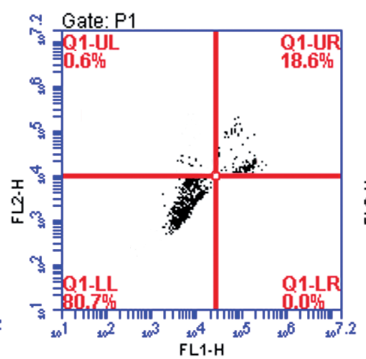

LPS $+50 \mu \mathrm{M}$ TSG

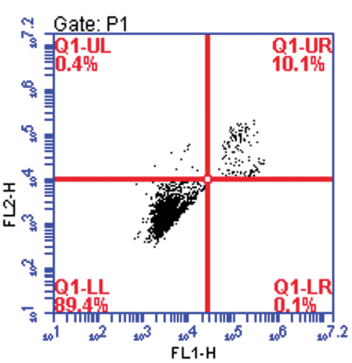

LPS $+100 \mu \mathrm{M} \mathrm{TSG}$
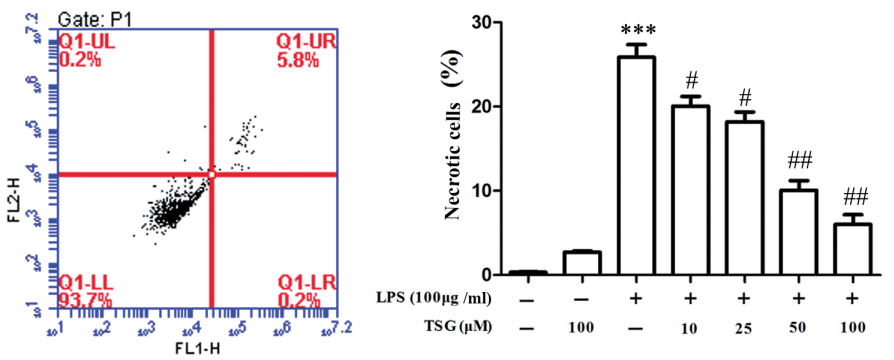

Figure 2. Protective effects of TSG on LPSinduced endothelial dysfunction. HUVECs were pretreated with different concentrations of TSG $(0,10,25,50$, and $100 \mu \mathrm{M})$ for $24 \mathrm{~h}$ before treatment with $100 \mu \mathrm{g} / \mathrm{ml}$ LPS for another $24 \mathrm{~h}$. A. Cell viability was assessed by MTT assay. B. LDH release assay in the supernatant culture medium. C. Cell apoptosis or necrosis was detected by flow cytometric analysis. The right bottom quadrant represents the annexin $\mathrm{V}$-stained cells (early-phase apoptotic), and the top right quadrant represents PI- and annexin V-stained cells (late-phase apoptotic/necrotic). Graphs shows the percentage of annexin $\mathrm{V}$ single positive and annexin V/PI double positive cells. Cells without treatment were the control group. Data are expressed as means $\pm \mathrm{SD}$ of three independent experiments. ${ }^{*} p<0.01$ and ${ }^{* * *} p<0.001 v s$. Control group; $\#<0.05$ and ${ }^{\# \#} p<0.01 v s$. LPS-treated group $(n=3)$. 
50 and $100 \mu \mathrm{M}$ TSG pretreatment significantly reduced the LDH release induced by LPS, while $100 \mu \mathrm{M}$ TSG treatment alone had no significant effects on the release of $\mathrm{LDH}$ in HUVECs. As illustrated in Fig. 2C, pretreatment with various concentrations of TSG $(10,25,50$, and $100 \mu \mathrm{M})$ significantly reduced the number of necrotic cells induced by LPS (IC50 = $39.76 \mu \mathrm{M})$, while TSG treatment alone ( $100 \mu \mathrm{M}$, for $24 \mathrm{~h})$ had no significant effects on the necrosis of normal HUVECs.

\section{Inhibitory effects of TSG on the RhoA/ROCK pathway induced by LPS}

To investigate whether the RhoA/ROCK pathway mediates LPS-induced HUVEC cell injury and whether TSG has an interventional effect on this pathway, we detected the expression of RhoA and ROCK, and the activation of ROCK by using Western blotting. As shown in Fig. 3A, compared with the control group, LPS significantly increased the expression of RhoA $(p<0.05)$, which was greatly inhibited by $100 \mu \mathrm{M}$ TSG pretreatment. The expression of RhoA in normal en- dothelial cells was not affected by $100 \mu \mathrm{M}$ TSG treatment alone. In addition, LPS significantly increased the expression of both ROCK1 and ROCK2 when compared with the control. TSG pretreatment at different concentrations (10, 25,50 , and $100 \mu \mathrm{M}$ ) dramatically decreased the expression of ROCK1 induced by LPS treatment, while LPS-induced ROCK2 expression was significantly inhibited only by $100 \mu \mathrm{M}$ TSG pretreatment (Fig. 3B). We did not detect any significant effects of $100 \mu \mathrm{M}$ TSG treatment alone on both ROCK1 and ROCK2 expression in normal HUVECs. We further examined the activation of ROCK induced by LPS by detecting the phosphorylation of myosin phosphatase targeting subunit 1 (MYPT1) (Arita et al. 2009). As illustrated in Fig. 3C, LPS treatment significantly enhanced the phosphorylation of MYPI1 (both at Thr696 and Thr853 sites) without affecting the total MYPT1 expression, which was greatly inhibited by pretreatment with 50 and $100 \mu \mathrm{M}$ TSG. In addition, $100 \mu \mathrm{M}$ TSG pretreatment alone had no significant effect on the total expression and phosphorylation of MYPT1 in normal endothelial cells. Therefore, these
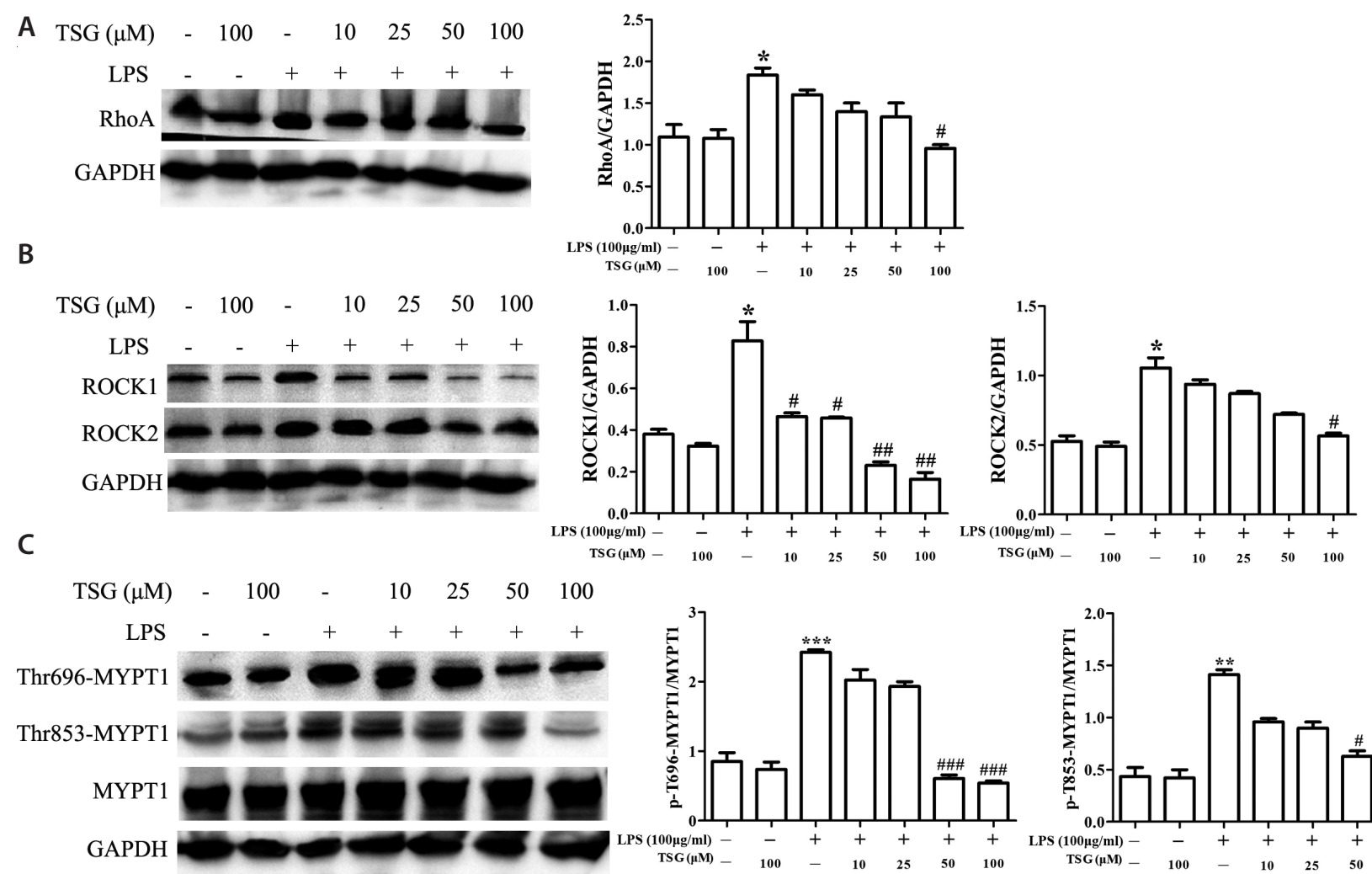

Figure 3. Effects of TSG on the LPS-induced RhoA/ROCK pathway. HUVECs were pretreated with different concentrations of TSG $(0,10$, 25, 50, and $100 \mu \mathrm{M}$ ) for $24 \mathrm{~h}$ before treatment with $100 \mu \mathrm{g} / \mathrm{ml}$ LPS for another $24 \mathrm{~h}$. A. Western blot analysis for RhoA expression. B. Western blot analysis of ROCK1 and ROCK2 expression. C. Western blot analysis of MYPT1 expression and phosphorylation (Thr696 and Thr853). The protein levels were quantified by densitometric analysis and normalized to GAPDH or total MYPT1. Histograms show the ratio of target proteins to GAPDH or MYPT1. Cells without treatment were Control group. Data are expressed as means \pm SD of three independent experiments. ${ }^{\star} p<0.05,{ }^{* *} p<0.01$ and ${ }^{* *} p<0.001 v$ s. Control group; ${ }^{\#} p<0.05,{ }^{\# \#} p<0.01$ and ${ }^{\# \# \#} p<0.001 v s$. LPS-treated group ( $n=3$ ). 
results demonstrate that the RhoA/ROCK pathway may be involved in LPS-induced endothelial cell injury and that TSG may inhibit LPS-induced endothelial dysfunction by inhibiting the RhoA/ROCK pathway.

\section{Effects of RhoA/ROCK pathway suppression on LPS-induced} endothelial dysfunction

To further confirm that the RhoA/ROCK signaling pathway is involved in LPS-induced endothelial cell toxicity, we first silenced the expression of RhoA, ROCK1, and ROCK2 in HUVECs by siRNA and then identified their expression by Western blot. The results showed that the transfection of RhoA-siRNA, ROCK1-siRNA, and ROCK2-siRNA successfully reduced the expression of RhoA, ROCK1, and ROCK2, respectively (Fig. 4A-C). MTT assays showed that the LPS-induced reduction of endothelial cell viability was significantly improved after the suppression of RhoA or ROCK or pretreatment with the ROCK inhibitor Y27632 (Fig. 4D). Furthermore,

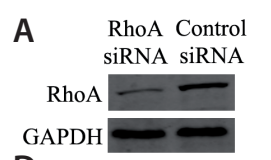

D

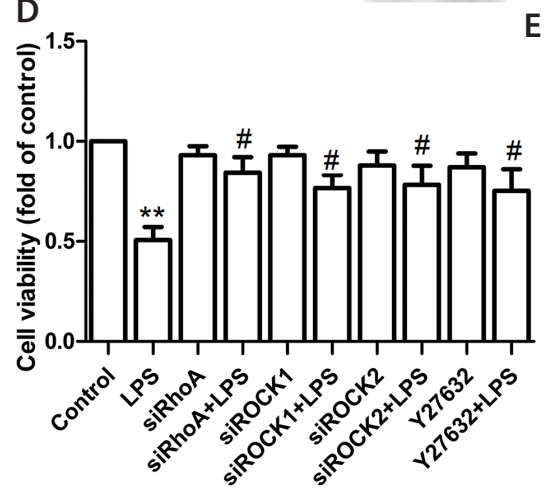

F

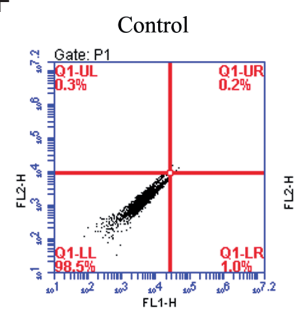

siROCK1
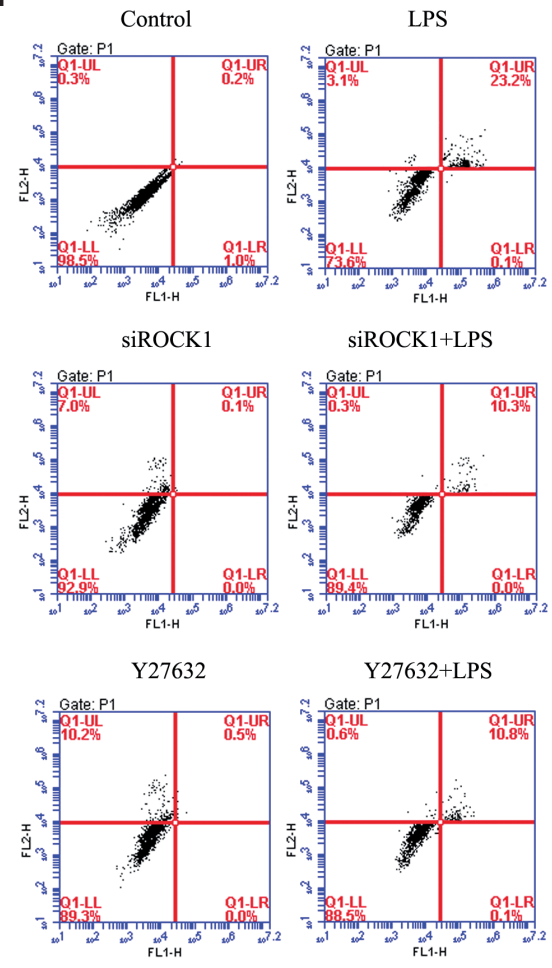

siROCK1+LPS
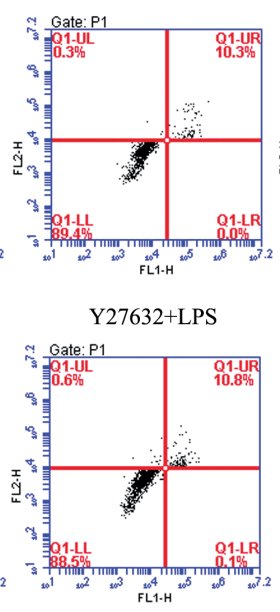

E
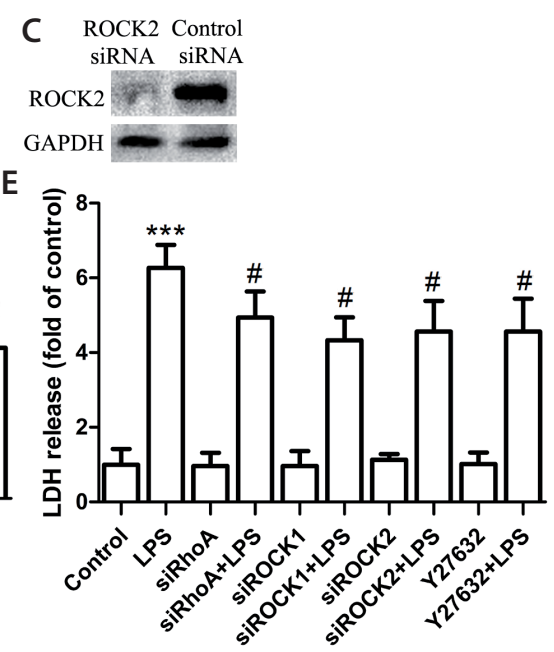

siRhoA

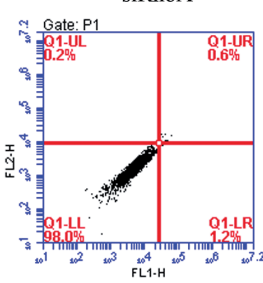

siROCK2

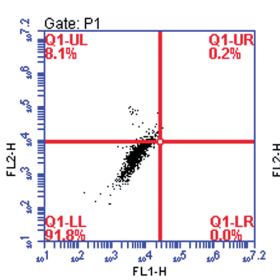

siROCK2+LPS

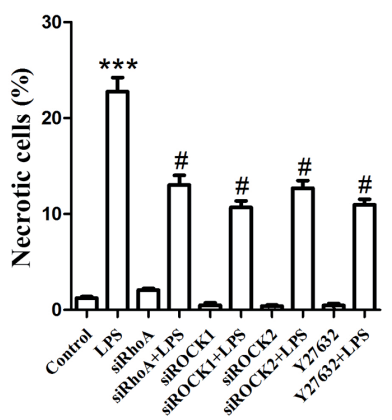

Figure 4. Effects of RhoA/ROCK pathway suppression on LPS-induced endothelial dysfunction. Cells were pretreated with $20 \mu \mathrm{M}$ Y27632 for $30 \mathrm{~min}$ before being incubated with $100 \mu \mathrm{g} / \mathrm{ml}$ LPS for $24 \mathrm{~h}$. The expression of RhoA was successfully inhibited by RhoA-siRNA transfection for $48 \mathrm{~h}$. A. Detection of RhoA suppression by Western blot analysis. B. Detection of ROCK1 suppression by western blot analysis. The expression of ROCK1 was successfully inhibited by ROCK1-siRNA transfection for 48 h. C. Detection of ROCK2 suppression by western blot analysis. The expression of ROCK2 was successfully inhibited by ROCK2-siRNA transfection for $48 \mathrm{~h}$. D. Cell viability was measured by MTT assay. E. LDH release assay in the supernatant culture medium. F. Cell apoptosis or necrosis was detected by flow cytometric analysis. The right bottom quadrant represents the annexin V-stained cells (early-phase apoptotic), and the top right quadrant represents PI- and annexin V-stained cells (late-phase apoptotic/necrotic). The histogram shows the percentage of annexin $\mathrm{V}$ single-positive and annexin V/PI double-positive cells. were the Data are expressed as means \pm SD of three independent experiments. ${ }^{* *} p<0.01$ and ${ }^{\star * *} p<0.001$ vs. Control group; ${ }^{\#} p<$ $0.05 v s$. LPS-treated group $(n=3)$. Control group, cells without treatment. 


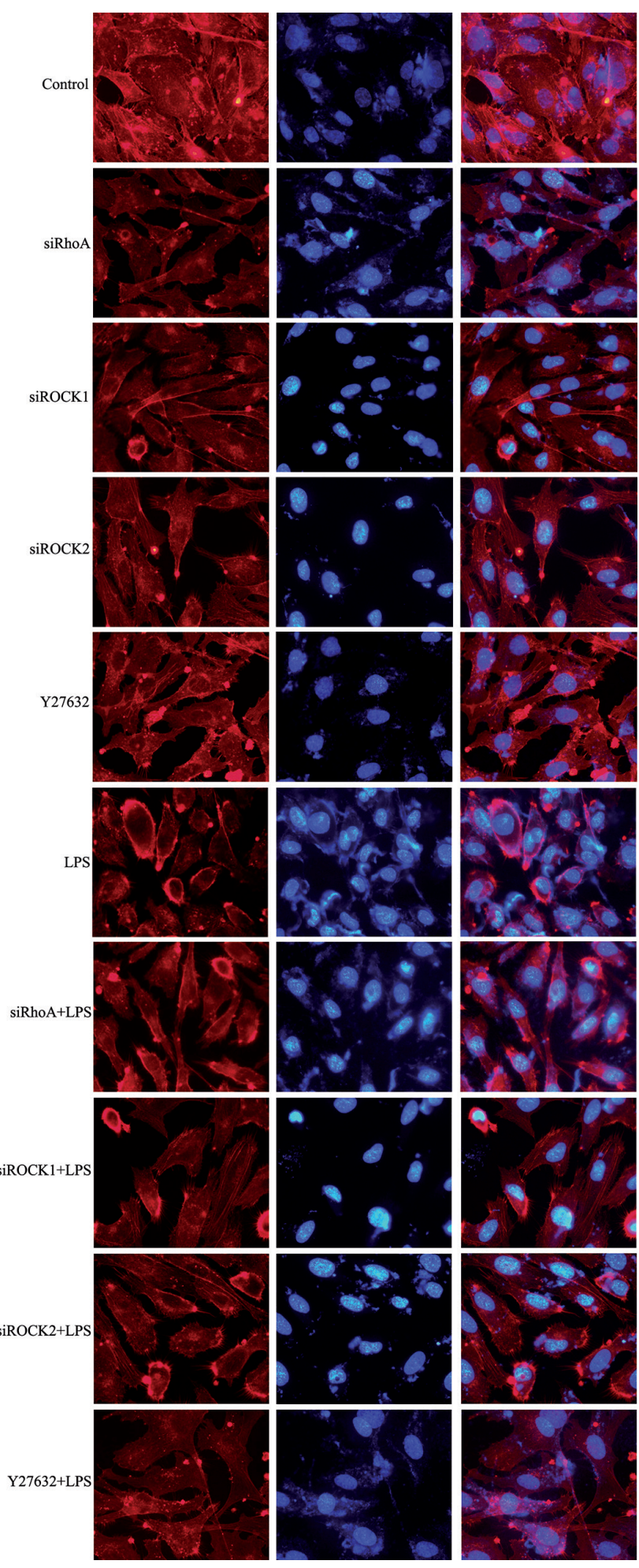

Figure 5. Phalloidin and phalloidin conjugates for staining F-actin filaments. Immunofluorescence assay for F-actin (red) and nuclei stained with DAPI (blue). Cells were pretreated with $20 \mu \mathrm{M}$ Y 27632 for $30 \mathrm{~min}$ before being incubated with $100 \mu \mathrm{g} / \mathrm{ml}$ LPS for $24 \mathrm{~h}$. RhoA or ROCK suppression inhibited the redistribution of F-actin induced by LPS. (See online version for color figure.)
LPS-induced LDH release was significantly inhibited by silencing the expression of RhoA or ROCK or pretreatment with Y27632 (Fig. 4E). And LPS-induced endothelial cell necrosis was also significantly decreased after the suppression of RhoA or ROCK or pretreatment with Y27632 (Fig. 4F). These results indicate that inhibition of the RhoA/ROCK signaling pathway can significantly reduce LPS-induced endothelial cell injury.

\section{Effects of RhoA/ROCK pathway suppression on LPS-in- duced F-actin remodeling}

It has been confirmed that the actin skeleton rearranges during cell apoptosis and motility activation (Amano et al. 1997; Shi et al. 2013). In this study, we observed the effects of LPS and RhoA/ROCK pathway suppression on the F-actin skeleton by performing phalloidin staining. As shown in Fig. 5, F-actin stained as red filaments was distributed over the whole cell in normal HUVECs. After treatment with LPS, cells were slightly contracted, and F-actin was mainly distributed around the plasma membrane to form a strong red fluorescence ring in the cell edge. This LPSinduced F-actin skeleton remodeling was inhibited by the suppression of RhoA, ROCK1, or ROCK2, or by pretreatment with Y27632 as shown by the decreased fluorescent rings around the plasma membrane and the increased distribution of F-actin over the whole cell. However, suppression of the RhoA/ROCK pathway exhibited no obvious effects on F-actin distribution in normal HUVECs. These results indicate that the suppression or inhibition of the RhoA/ROCK signaling pathway can reverse LPS-induced F-actin cytoskeleton remodeling.

\section{Effects of TSG on LPS-induced F-actin remodeling}

Furthermore, we observed the effects of TSG on the remodeling of F-actin induced by LPS. As shown in Fig. 6, 50 and $100 \mu \mathrm{M}$ TSG pretreatment dramatically decreased the red fluorescent rings in the cell edges induced by LPS and reversed the distribution of F-actin throughout the cells. However, pretreatment with $100 \mu \mathrm{M}$ TSG alone did not exhibit significant effects on F-actin distribution in normal endothelial cells. These results demonstrate that TSG treatment can inhibit LPS-induced F-actin cytoskeleton remodeling.

\section{Discussion}

The RhoA/ROCK signaling pathway has been reported to be involved in multiple diseases, such as diabetic nephropathy, cancer, and hypertension. RhoA/ROCK signaling induces cytoskeletal reorganization and stresses fiber formation, which participates in many physiological functions (Riento 
and Ridley 2003; Arita et al. 2009; Satoh et al. 2011). In this study, we first elucidated the regulation of the RhoA/ROCK signaling pathway in LPS-induced endothelial injury and F-actin cytoskeleton remodeling.

We found that LPS treatment significantly reduced the survival rate of endothelial cells, and increased the release of $\mathrm{LDH}$ and cell necrosis in a dose- and time-dependent manner. The viability of cells exposed to 25,50 , and $100 \mu \mathrm{g} / \mathrm{ml}$ of LPS decreased to $0.75 \pm 0.08,0.61 \pm 0.06$, and $0.52 \pm 0.09$ compared with the control, respectively. Since the survival rate of cells treated with $100 \mu \mathrm{g} / \mathrm{ml}$ LPS was greater than $50 \%$, we thus selected $100 \mu \mathrm{g} / \mathrm{ml}$ LPS treatment for further experiments in this study. Since small Rho GTPases act as molecular switches in a range of cellular activities (Bishop and Hall 2000; Heasman and Ridley 2008), we observed the effects of LPS on the expression of RhoA. The results showed that LPS induction significantly enhanced the expression of RhoA. The suppression of RhoA by siRNA significantly improved the endothelial cell survival rate and reduced LPS-mediated endothelial cell necrosis and $\mathrm{LDH}$ release, indicating that RhoA is indeed involved in LPS-induced endothelial cell injury. ROCK, a serine/threonine protein kinase, is an important effector protein of RhoA and includes two subtypes, ROCK1 and ROCK2 (Yao et al. 2013). Our results illustrated that LPS treatment significantly induced the expression of both ROCK1 and ROCK2 as well as the activation of ROCK. After successfully suppressing the expression of ROCK1 and ROCK2 or pretreating with the non-selective ROCK inhibitor Y-27632 (which equivalently inhibits ROCK1 and ROCK2), cell necrosis and LDH release induced by LPS were significantly inhibited, and the endothelial cell survival rate was dramatically improved. These results indicate that the RhoA/ROCK signaling pathway participates in LPS-induced endothelial cytotoxicity.

It has been reported that the reorganization of F-actin in endothelial cells is an important pathological factor leading to increased endothelial permeability and endothelial dysfunction (Shi et al. 2013; Wang et al. 2013). It has been found that activated RhoA regulates the phosphorylation of the downstream myosin light chain (MLC) by activating ROCK, causing the formation of stress fibers and focal adhesion and the remodeling of F-actin (Shi et al. 2013; Wang et al. 2013). To find out whether the F-actin cytoskeleton regulated by the ROCK pathway plays a role in LPS-induced endothelial cell injury, we examined the effects of the RhoA/ROCK pathway on LPS-mediated F-actin remodeling. We found that LPS treatment promoted F-actin skeleton rearrangement and the formation of contraction rings around the plasma membrane, accompanied by cell morphology changes, which were greatly inhibited by suppression of the RhoA/ROCK pathway. These results indicate that the regulation of the Factin cytoskeleton by the RhoA/ROCK signaling pathway is involved in LPS-induced endothelial cell injury.
It has been reported that TSG has significant pharmacological activities such as antioxidant and anti-inflammatory activities (Xu et al. 2012; Zhang et al. 2012; Büchter et al. 2015; Chen et al. 2017). In this study, we found that TSG significantly inhibited LPS-induced LDH release and cell necrosis, and improved endothelial cell viability. In addition, TSG significantly suppressed the expression of RhoA, ROCK1, ROCK2, and the activation of ROCK induced by LPS. We also found that TSG inhibited LPS-induced F-actin cytoskeleton remodeling and reduced the formation of contracting rings and cell morphological changes. Taken

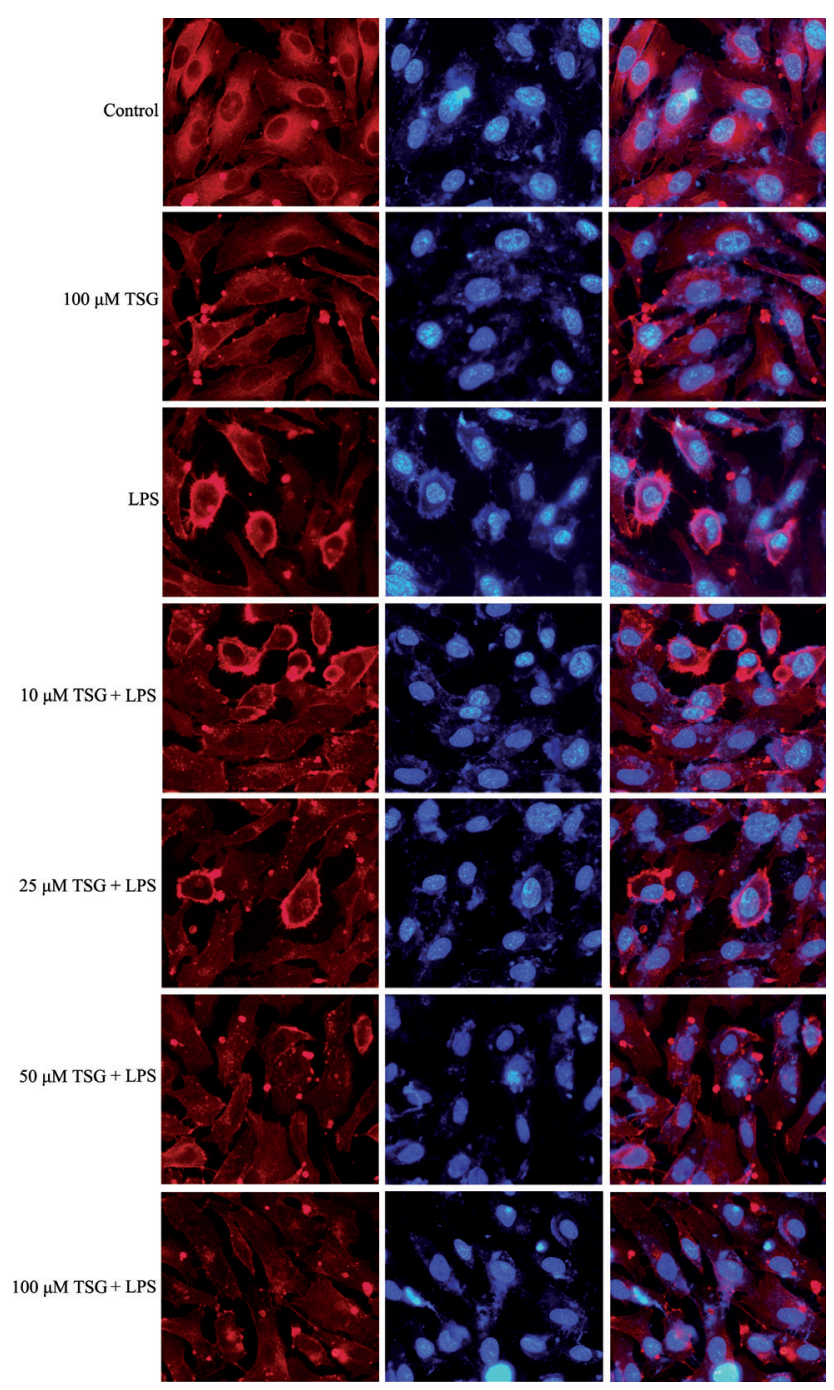

Figure 6. Phalloidin and phalloidin conjugates for staining actin filaments. Immunofluorescence assay for F-actin (red) and nuclei stained with DAPI (blue). Cells were pretreated with different concentrations of TSG $(10,25,50$, and $100 \mu \mathrm{M})$ for $24 \mathrm{~h}$ and then treated with $100 \mu \mathrm{g} / \mathrm{ml}$ LPS for another $24 \mathrm{~h}$. TSG pretreatment inhibited the redistribution of F-actin induced by LPS. (See online version for color figure.) 
together, TSG may regulate F-actin cytoskeletal rearrangements by inhibiting the RhoA/ROCK signaling pathway and thereby reduces LPS-induced endothelial cell toxicity.

In conclusion, the main findings of our study are: (i) the RhoA/ROCK signaling pathway is involved in LPSinduced endothelial cytotoxicity by regulating the F-actin cytoskeleton; (ii) TSG reduces LPS-induced endothelial cell toxicity by inhibiting RhoA/ROCK signaling and F-actin cytoskeleton remodeling.

Acknowledgements. This work was supported by a grant from the National Natural Sciences Foundation of China (81970226), Key University Science Research Project of Jiangsu Province (19KJA320010), the Natural Science Foundation of Jiangsu Province (BK20180267).

Contributions. YQ and WY conceived the study, and participated in its design together. $\mathrm{XH}$ and $\mathrm{HH}$ participated in the cell culture and treatment. YQ, XL, and LT participated in MTT assay, LDH release assay, Western blotting and Flow cytometry analysis. XL performed siRNA transfection, F-actin imaging, and the statistical analysis. YQ wrote the initial manuscript, XL helped to revise it critically, both of them are co-first authors. WY is the corresponding author. All authors read and approved the final manuscript.

Conflict of interest. The authors declared no potential conflicts of interest with respect to the research, authorship, and/or publication of this article.

\section{References}

Amano M, Chihara K, Kimura K, Fukata Y, Nakamura N, Matsuura Y, Kaibuchi K (1997): Formation of actin stress fiber and focal adhesions enhanced by Rho-kinase. Science 275, 1308-1311 https://doi.org/10.1126/science.275.5304.1308

Arita R, Hata Y, Nakao S, Kita T, Miura M, Kawahara S, Zandi S, Almulki L, Tayyari F, Shimokawa H, et al. (2009): Rho kinase inhibition by fasudil ameliorates diabetes-induced microvascular damage. Diabetes 58, 215-226 https://doi.org/10.2337/db08-0762

Bishop AL, Hall A (2000): Rho GTPases and their effector proteins. Biochem. J. 348, 241-255 https://doi.org/10.1042/bj3480241

Büchter C, Zhao L, Havermann S, Honnen S, Fritz G, Proksch P, Wätjen W (2015): TSG (2,3,5,4'-Tetrahydroxystilbene-2-O- $\beta$ $\mathrm{D}$-glucoside) from the Chinese herb polygonum multiflorum increases life span and stress resistance of caenorhabditis elegans. Oxid. Med. Cell. Longev. 2015, 124357 https://doi.org/10.1155/2015/124357

Charbonney E, Tsang JY, Li Y, Klein D, Duque P, Romaschin A, Marshall JC (2016): Endotoxemia following multiple trauma: risk factors and prognostic implications. Crit. Care. Med. 44, 335-341

https://doi.org/10.1097/CCM.0000000000001404
Chen X, Hu W, Lu X, Jiang B, Wang J, Zhang W, Huang C (2017): Mechanism of 2,3,4,5-tetrahydroxystilbene 2-O- $\beta$-Dglucoside-induced upregulation of glutamate transporter 1 protein expression in mouse primary astrocytes. Pharmacology $99,153-159$ https://doi.org/10.1159/000452672

Cheng KT, Xiong S, Ye Z, Hong Z, Di A, Tsang KM, Gao X, An S, Mittal M, Vogel SM, et al. (2017): Caspase-11-mediated endothelial pyroptosis underlies endotoxemia-induced lung injury. J. Clin. Invest. 127, 4124-4135 https://doi.org/10.1172/JCI94495

Fu P, Usatyuk PV, Lele A, Harijith A, Gregorio CC, Garcia JG, Salgia R, Natarajan V (2015): c-Abl mediated tyrosine phosphorylation of paxillin regulates LPS-induced endothelial dysfunction and lung injury. Am. J. Physiol. Lung Cell Mol. Physiol. 308, 1025-1038

https://doi.org/10.1152/ajplung.00306.2014

Han X, Ling S, Gan W, Sun L, Duan J, Xu JW (2012): 2,3,5,4'-tetrahydroxystilbene- 2-O-beta-d-glucoside ameliorates vascular senescence and improves blood flow involving a mechanism of p53 deacetylation. Atherosclerosis 225, 76-82 https://doi.org/10.1016/j.atherosclerosis.2012.08.011

Heasman SJ, Ridley AJ (2008): Mammalian Rho GTPases: new insights into their functions from in vivo studies. Nat. Rev. Mol. Cell. Biol. 9, 690-701 https://doi.org/10.1038/nrm2476

Matsui T, Amano M, Yamamoto T, Chihara K, Nakafuku M, Ito M, Nakano T, Okawa K, Iwamatsu A, Kaibuchi K (1996): Rho-associated kinase, a novel serine/threonine kinase, as a putative target for small GTP binding protein Rho. EMBO J. 15, 2208-2216 https://doi.org/10.1002/j.1460-2075.1996.tb00574.x

Nakagawa O, Fujisawa K, Ishizaki T, Saito Y, Nakao K, Narumiya S (1996): Rock-i and rock-ii, two isoforms of Rho-associated coiled-coil forming protein serine/threonine kinase in mice. FEBS Lett. 392, 189-193 https://doi.org/10.1016/0014-5793(96)00811-3

Riento K, Ridley AJ (2003): Rocks: multifunctional kinases in cell behavior. Nat. Rev. Mol. Cell. Biol. 4, 446-456 https://doi.org/10.1038/nrm1128

Satoh K, Fukumoto Y, Shimokawa H (2011): Rho-kinase: important new therapeutic target in cardiovascular diseases. Am. J. Physiol. Heart. Circ. Physiol. 301, H287-296 https://doi.org/10.1152/ajpheart.00327.2011

Shi J, Wu X, Surma M, Vemula S, Zhang L, Yang Y, Kapur R, Wei L (2013): Distinct roles for ROCK1 and ROCK2 in the regulation of cell detachment. Cell Death Dis. 4, e483 https://doi.org/10.1038/cddis.2013.10

Straat M, Tuip A, Klei TRL, Beuger BM, Roelofs JJTH, Bruggen RV, Juffermans NP (2017): Endotoxemia results in trapping of transfused red blood cells in lungs with associated lung injury. Shock 48, 484-489 https://doi.org/10.1097/SHK.0000000000000875

Tian R, Li R, Liu Y, Liu J, Pan T, Zhang R, Liu B, Chen E, Tang Y, Qu H (2019): Metformin ameliorates endotoxemia-induced endothelial pro-inflammatory responses via AMPK-dependent mediation of HDAC5 and KLF2. Biochim. Biophys. Acta 1865, 1701-1712 https://doi.org/10.1016/j.bbadis.2019.04.009 
Wang CH, Wu CC, Hsu SH, Liou JY, Li YW, Wu KK, Lai YK, Yen BL (2013): The role of RhoA kinase inhibition in human placenta-derived multipotent cells on neural phenotype and cell survival. Biomaterials 34, 3223-3230 https://doi.org/10.1016/j.biomaterials.2012.12.034

Wu J, Hu W, Gong Y, Wang P, Tong L, Chen X, Chen Z, Xu X, Yao W, Zhang W, Huang C (2017): Current pharmacological developments in 2,3,4,5-tetrahydroxystilbene 2-O- $\beta$-D-glucoside (TSG). Eur. J. Pharmacol. 811, 21-29 https://doi.org/10.1016/j.ejphar.2017.05.037

Xu XL, Huang YJ, Chen XF, Lin DY, Zhang W (2012): $2,3,4^{`}, 5$-tetrahydroxystilbene-2-O- $\beta$-D-glucoside inhibits proliferation of vascular smooth muscle cells: involvement of NO/cGMP/PKG pathway. Phytother. Res. 6, 1068-1074 https://doi.org/10.1002/ptr.3691

Xu Z, Wu G, Wei X, Chen X, Wang Y, Chen L (2013): Celastrol induced DNA damage, cell cycle arrest, and apoptosis in human rheumatoid fibroblast-like synovial cells. Am. J. Chin. Med. 41, 615-628

https://doi.org/10.1142/S0192415X13500432
Yao L, Chandra S, Toque HA, Bhatta A, Rojas M, Caldwell RB, Caldwell RW (2013): Prevention of diabetes-induced arginase activation and vascular dysfunction by Rho kinase (ROCK) knockout. Cardiovasc. Res. 97, 509-519 https://doi.org/10.1093/cvr/cvs371

Zhang W, Wang CH, Li F, Zhu WZ (2008): 2,3,4,5-Tetrahydroxystilbene-2-O-beta-D-glucoside suppresses matrix metalloproteinase expression and inflammation in atherosclerotic rats. Clin. Exp. Pharmacol. Physiol. 35, 310-316 https://doi.org/10.1111/j.1440-1681.2007.04824.x

Zhang W, Chen XF, Huang YJ, Chen QQ, Bao YJ, Zhu W (2012): $2,3,4,5$-Tetrahydroxystilbene-2-O- $\beta$-D-glucoside inhibits angiotensin II-induced cardiac fibroblast proliferation via suppression of the reactive oxygen species-extracellular signalregulated kinase 1/2 pathway. Clin. Exp. Pharmacol. Physiol. 39, 429-437

https://doi.org/10.1111/j.1440-1681.2012.05692.x

Received: March 16, 2020

Final version accepted: July 16, 2020 